\title{
CMB Signatures of a Primordial Magnetic Field
}

\author{
Tina Kahniashvili ${ }^{1,2 \dagger}$, Arthur Kosowsky ${ }^{2, \ddagger}$, Andrew Mack ${ }^{2, \ddagger}$, and \\ Ruth Durrer ${ }^{3, \dagger}$ \\ ${ }^{1}$ Center for Plasma Astrophysics, Abastumani Astrophysical Observatory, Kazbegi Ave. 2a, \\ 380060 Tbilisi, Georgia \\ ${ }^{2}$ Dept. of Physics and Astronomy, Rutgers University, 136 Frelinghuysen Road, Piscataway, NJ \\ 08854 USA \\ ${ }^{3}$ Université de Genève, Département de Physique Théorique, Switzerland \\ ${ }_{\dagger}^{\dagger}$ tinatin,durrer@amorgos.unige.ch, ${ }^{\ddagger}$ kosowsky, andymack@physics.rutgers.edu
}

\begin{abstract}
A primordial stochastic magnetic field will induce temperature and polarization fluctuations in the cosmic microwave background. We outline a calculation of the resulting fluctuation power spectra and present numerical results.
\end{abstract}

\section{INTRODUCTION}

The presence of a magnetic field in the early universe influences the evolution of metric perturbations, and as a result, might leave observable traces in the cosmic microwave background (CMB). During the past few years the gravitational effects of a homogeneous magnetic field were presented by several authors; for a detailed review see [1] and references therein. More realistic is the case of a stochastic magnetic field, because any causal generation mechanism results in stochastic fields. Some estimates of CMB temperature power spectra from density perturbations induced by a stochastic magnetic field are given in [2] and numerical simulations are presented in [3]. The vorticity induced by a stochastic magnetic field was studied in [4] and CMB temperature fluctuations were obtained in the case of a single $k$ mode. The gravitational waves generated by tangled magnetic fields and the resulting tensor CMB temperature fluctuations spectra are given in [5].

Here we outline a comprehensive analytic calculation of temperature and polarization power spectra due to all types of metric perturbations arising from a primordial stochastic magnetic field, which we assume to be statistically homogeneous and isotropic and described by a power law. Detailed results will be presented elsewhere. 


\section{METRIC PERTURBATIONS INDUCED BY MAGNETIC FIELDS}

The energy density of the magnetic field is treated as a first order perturbation, which thus does not alter the background cosmology. Prior to decoupling, the conductivity of the primordial plasma can be assumed to be infinite, which in the comoving frame implies the "frozen-in" condition $\mathbf{E}=-\mathbf{v} \times \mathbf{B}$, where $\mathbf{v}$ is the plasma velocity and $\mathbf{E}$ is the electric field induced by plasma motions. Infinite conductivity leads to a vanishing electric field in linear perturbations theory $(v \ll 1)$ and permits a split of the spatial structure and time dependence of the magnetic field. As the universe expands, magnetic field lines are simply conformally diluted due to flux conservation: $\mathbf{B}(\eta, \mathbf{x})=\mathbf{B}\left(\eta_{0}, \mathbf{x}\right) / a^{2}$, where $a$ is the scale factor.

We assume that magnetic field is distributed homogeneously and isotropically with a power law power spectrum [5]:

$$
\left\langle B_{i}(\mathbf{k}) B_{j}^{*}\left(\mathbf{k}^{\prime}\right)\right\rangle=(2 \pi)^{5} \frac{\lambda^{n+3}}{\Gamma\left(\frac{n+3}{2}\right)} B_{\lambda}^{2}\left(\delta_{i j}-\hat{k}_{i} \hat{k}_{j}\right) k^{n} \delta\left(\mathbf{k}-\mathbf{k}^{\prime}\right), \quad k<k_{D} .
$$

The spectrum vanishes for all scales smaller than a damping scale $k>k_{D}$; we require the spectral index $n>-3$ so as not to overproduce superhorizon coherent fields. $B_{\lambda}$ is the rms magnetic field strength today smoothed over a co-moving length scale $\lambda$.

The electromagnetic stress-energy tensor may be geometrically decomposed into scalar (density), vector (vorticity) and tensor (gravitational wave) perturbation modes: $\tau_{i j}^{(B)}=\Pi_{i j}^{(S)}+\Pi_{i j}^{(V)}+\Pi_{i j}^{(T)}$. From the tensor $\Pi_{i j}^{(S)}$ we can construct scalar $\Pi^{(S)}$ which describes the density perturbations, and from the tensor $\Pi_{i j}^{(V)}$ a vector $\Pi_{i}^{(V)}$ which describes the vorticity perturbations. Then $\Pi^{(S)}$ is proportional to the magnetic field energy density $\left|\rho_{B}\left(\mathbf{k}, \eta_{0}\right)\right| \simeq \frac{3}{2}\left|\Pi^{(S)}\left(\mathbf{k}, \eta_{0}\right)\right|$, and $\Pi_{i}^{(V)}$ is proportional to the vortical piece of the Lorentz force, $L_{i}^{(V)}=k \Pi_{i}^{(V)}$. The isotropic spectra $\Pi^{(S),(V),(T)}$ appear as source terms for scalar, vector and tensor perturbations (for details see [5], [7]).

To obtain the evolution equations for perturbations, we must derive the scalar, vector and tensor magnetic field correlation functions for $\Pi^{(S)}, \Pi_{i}^{(V)}$ and $\Pi_{i j}^{(T)}$ as described in [5] and obtain the corresponding spectra as a functions of $n, B_{\lambda}$ and $k_{D}$. Terms induced by the magnetic field (e.g. Lorentz force, magnetic field anisotropic stresses) appear only in the dynamical equations describing baryons. We use notational conventions of Ref. [6] and employ the Newtonian gauge; $a$ is the scale factor, $\eta$ is conformal time, and 0 subscripts refer to the present time.

Scalar perturbations: Since we assume that the background spacetime is unperturbed and linear order perturbations arise only from the magnetic field, the stress energy of the magnetic field is not compensated by anisotropic stress of the plasma and we have non-zero initial conditions for gravitational potentials. Such initial conditions might arise from an inflation-like process; the compensated case, arising 
from causal processes like phase transitions, has more complicated dynamical effects, but our estimates should still be of the correct order of magnitude. We limit the spectral index of the magnetic field to $n<2$ [5]; the case $n>2$ corresponds to causally generated fields, which also must be compensated.

The scalar constraint equations from the Einstein equations are two Poisson equations,

$$
k^{2} \Phi=4 \pi G a^{2}\left[\rho_{f} \delta_{f}+\rho_{B}\right], \quad \quad k^{2}(\Psi+\Phi)=-12 \pi G a^{2} \Pi^{(S)},
$$

where $\rho_{f} \delta_{f}$ is the total fluid perturbation. The dynamical Einstein equations determine the evolution of the photon and baryon densities (see also [8]):

$$
\dot{\delta}_{\gamma}=-\frac{4}{3}\left(k v_{\gamma}^{(S)}+3 \dot{\Phi}\right), \quad \dot{\delta}_{\mathrm{cdm}}=-\left(k v_{\mathrm{cdm}}^{(S)}+3 \dot{\Phi}\right), \quad \dot{\delta}_{b}=-\left(k v_{b}^{(S)}+3 \dot{\Phi}\right)-3 \frac{\dot{a} \frac{\Pi^{(S)}}{a}}{\rho_{b}} .
$$

Using the zero-order approximation for tight coupling regime, $v_{b} \simeq v_{\gamma} \simeq v^{(S)}$, we obtain the following equation of motion [7] from momentum conservation equations for baryons and photons:

$$
\dot{v}^{(S)}-\frac{k}{4} \delta_{\gamma}-k \Psi+\frac{k \Pi^{(S)}}{\rho_{\gamma}+p_{\gamma}}=0 .
$$

Using the equations of motion, it is possible to show that the two terms on the right side of the first Poisson equation are roughly equal. An approximate solution for the gravitational potential is then

$$
\Phi(\eta) \simeq \frac{12 \pi G \Pi^{(S)}\left(\eta_{0}, k\right)}{k^{2} a^{2}}
$$

Vector perturbations: The vector Einstein equation which describes the evolution of the vector potential sourced by a stochastic magnetic field is

$$
\dot{V}_{i}+2 \frac{\dot{a}}{a} V_{i}=-16 \pi G a^{2} \Pi_{i}^{(V)}(\eta, \mathbf{k}) / k .
$$

Another constraint equation relates the vector potential to the vorticity $\Omega$ :

$$
-k^{2} V_{i}=16 \pi G a^{2}(\rho+p) \Omega_{i} .
$$

Introducing the Lorentz force term into the baryon Euler equation, neglecting baryon inertia, and solving the baryon momentum conservation equation in the tight-coupling approximation, we obtain an approximate solution for the vorticity,

$$
\boldsymbol{\Omega}(\eta, \mathbf{k}) \simeq \frac{k \boldsymbol{\Pi}^{(V)}\left(\eta_{0}, \mathbf{k}\right) \eta}{\rho_{\gamma 0}+p_{\gamma 0}}
$$

Tensor perturbations: The tensor Einstein equation that describes the evolution of gravitational waves sourced by a stochastic magnetic field is $[2,5]$ 


$$
\ddot{H}_{i j}+2 \frac{\dot{a}}{a} \dot{H}_{i j}+k^{2} H_{i j}=8 \pi G a^{2} \Pi_{i j}^{(T)}(\eta, \mathbf{k}) .
$$

The tensor case has no additional constraint equations. As in the case of vector perturbations, we neglect the tensor anisotropic stress of the plasma, which is in general negligible. A Green function technique gives an approximate solution for $\eta>\eta_{\text {eq }}[5]$

$$
\dot{H}(\eta, k) \simeq 4 \pi G \eta_{0}^{2} z_{\mathrm{eq}} \ln \left(\frac{z_{\text {in }}}{z_{\text {eq }}}\right) k \Pi^{(T)}\left(\eta_{0}, k\right) \frac{j_{2}(k \eta)}{k \eta},
$$

where $H(\eta, k)$ and $\Pi^{(T)}\left(\eta_{0}, k\right)$ are isotropic correlation functions of the tensors $H_{i j}$ and $\Pi_{i j}^{(T)}$ respectively, $z_{\text {in }}$ is the initial redshift at which the magnetic field is generated, and $z_{\text {eq }}$ is the redshift at matter-radiation equality.

\section{CMB ANISOTROPIES}

The solutions for the metric perturbations presented above can be used to compute the power spectra of temperature and polarization anisotropies in the CMB. The contributions from scalar, vector, and tensor perturbations are all uncorrelated. For all three cases, the temperature fluctuations arise simply from the Sachs-Wolfe effect, while the polarization fluctuations are sourced by these temperature fluctuations. For the scalar case, the temperature perturbation is just the familiar $-\Phi / 3$ at decoupling. The vector and tensor cases are slightly less familiar; expressions are given in Ref. [9] for vector perturbations and Ref. [5] for tensor perturbations.

We have derived analytic approximations to the angular power spectra of temperature and polarization for all three types of metric perturbation, neglecting small-scale damping effects due to photon diffusion or magnetic damping. As examples, for scalar perturbations with $n<-3 / 2$, the temperature power spectrum is

$\ell^{2} C_{\ell}^{T T} \simeq \frac{(2 \pi)^{5}}{3} \frac{G^{2} \eta_{0}^{4}}{a_{\mathrm{dec}}^{4}} \frac{2^{2 n+1} n}{(n+3)(2 n+3)} \frac{\Gamma(-2 n)}{\Gamma^{2}\left(-n+\frac{1}{2}\right) \Gamma^{2}\left(\frac{n+3}{2}\right)}\left(\frac{\lambda}{\eta_{0}}\right)^{2 n+6} \ell^{2 n+2} B_{\lambda}^{4}$,

while the power spectrum for the polarization-temperature cross correlation is

$$
\ell^{2} C_{\ell}^{T E} \simeq-\frac{8 \pi^{4}}{9} \frac{G \eta_{0}^{2}}{\rho_{\gamma 0}+p_{\gamma 0}} \frac{2^{2 n-1} n}{(n+3)(2 n+3)} \frac{\Gamma(-2 n-2)}{\Gamma^{2}\left(-n-\frac{1}{2}\right) \Gamma^{2}\left(\frac{n+3}{2}\right)}\left(\frac{\lambda \ell}{\eta_{0}}\right)^{2 n+6}\left(\frac{\eta_{0}}{\eta_{\mathrm{dec}}}\right)^{2} B_{\lambda}^{4} .
$$

Complete expressions for all cases will be presented elsewhere; only the temperature power spectrum for tensors [5] and a partial calculation of the temperature power spectrum for vectors [4] have been worked out previously. 


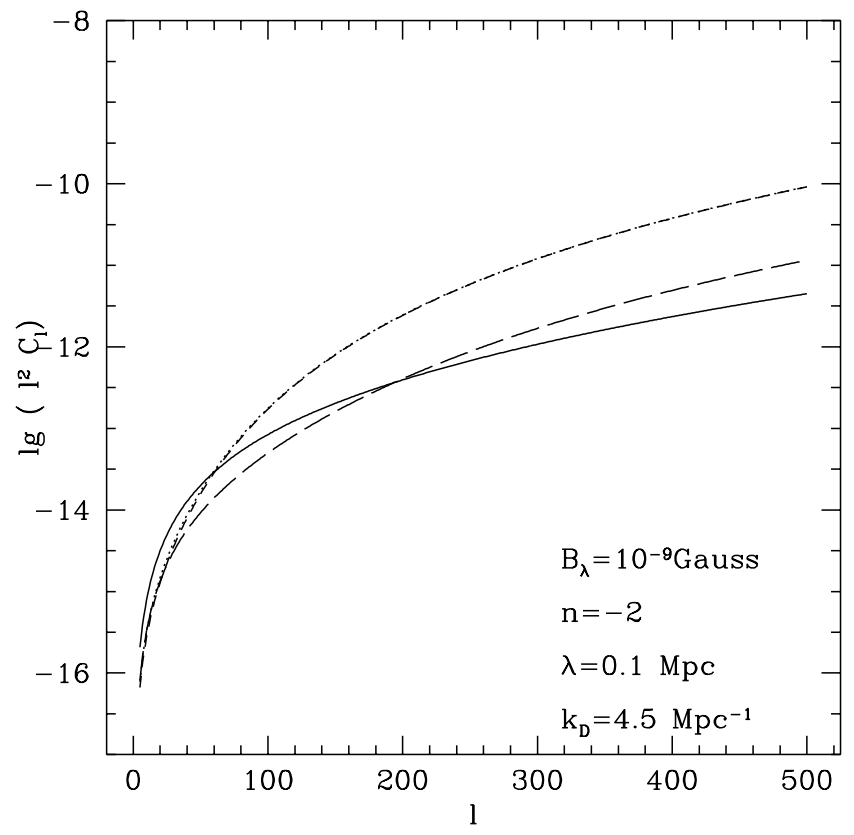

FIGURE 1. The total power spectra $\ell^{2} C_{\ell}$ vs. $\ell$ for $n=-2$ are shown: $\ell^{2} C_{\ell}^{T T}$ - solid line, $\ell^{2} C_{\ell}^{E E}$ - dotted line, $\ell^{2} C_{\ell}^{B B}$ - short dashed line, $\ell^{2} C_{\ell}^{T E}$ long-dashed line.

The total power spectra for $n=-2$ are shown in the Figure. For the smoothing scale we take $\lambda=0.1 \mathrm{Mpc}$, which corresponds to the maximum comoving length scale of galaxies (as in [5]); for the magnetic field damping scale we take the Alfven cut-off scale $[4,5]$, which is roughly $k_{D} \simeq 4.5 h \mathrm{Mpc}^{-1}$. Photon diffusion and magnetic damping will give an exponential cutoff to this fluctuation power spectra above a characteristic value of $\ell$. Note that for $n>-2$, the polarization power spectra are actually larger than the temperature power spectrum for $l>50$.

The magnetic field spectral index is confined to the range $-3<n<2$. As shown in $[5,7]$, no spectral index will produce a scale-invariant CMB power spectrum $\left(\ell^{2} C_{\ell}\right.$ constant) for scalar temperature and polarization anisotropies while $n \rightarrow-3$ results in a scale-invariant cross correlation spectrum $\ell^{2} C_{\ell}^{T E}$. The vector perturbations cannot produce scale-invariant spectra for allowed values of $n$; for tensor perturbations both, the temperature and polarization spectra $\ell^{2} C_{\ell}$ are scaleinvariant for $n \rightarrow-3$. Scalar perturbations are always subdominant if compared to vector and the dominant tensor perturbations.

The CMB power spectra due to a magnetic field vary in amplitude like the square of the energy density perturbations, or as $B^{4}$. It will thus be impractical to obtain upper limits on magnetic field strengths which are significantly more stringent than around $10^{-9}$ Gauss; limits improve as $n$ decreases. On the other hand, if primordial fields are present at this level, the combined signature in the various microwave background power spectra will give an unmistakable signal. We also note that the 
vector and tensor perturbations generated by magnetic fields are one of the few cosmological sources of "B" polarization [10], along with primordial tensor perturbations and gravitational lensing of the microwave background. Stochastic magnetic fields also will induce Faraday rotation in the microwave background polarization, providing an additional "B" polarization signal; we are currently considering this question.

Acknowledgments: This work has been supported by the COBASE program of the U.S. National Research Council and by the NASA Theory Program through grant NAG5-7015. T.K. acknowledges a grant from the Swiss NSF for participation in CAPP2000. A.K. is a Cotrell Scholar of the Research Corporation.

\section{REFERENCES}

1. D. Grasso and H. Rubinstein, astro-ph/0009061 (2000).

2. D. Lemoine, Cosmic Microwave Background Anisotropies Generated by a Primordial Magnetic Field, unpublished (1995).

3. S. Koh and C.H. Lee, astro-ph/0006357.

4. K. Subramanian and J. D. Barrow, Phys. Rev. Lett. 81, 3575 (1998).

5. R. Durrer, P.G. Ferreira and T. Kahniashvili, Phys. Rev. D 61, 043001 (2000).

6. W. Hu and M. White, Phys. Rev. D 57, 596 (1997).

7. T. Kahniashvili, A. Kosowsky, A. Mack, 2000, in preparation.

8. J. Adams, U. H. Danielsson, D. Grasso, and H. Rubinstein, Phys. Lett. B 388, 253 (1996).

9. R. Durrer, Fundam. Cosm. Phys. 15, 209 (1994).

10. M. Kamionkowski, A. Kosowsky, and A. Stebbins, Phys. Rev. Lett. 78, 2058 (1997). 